\title{
IL-17A and IL-17F do not contribute vitally to autoimmune neuro-inflammation in mice
}

\author{
Stefan Haak,, ${ }^{1}$ Andrew L. Croxford, ${ }^{2}$ Katharina Kreymborg, ${ }^{1}$ Frank L. Heppner, ${ }^{3}$ Sandrine Pouly, ${ }^{4}$ \\ Burkhard Becher, ${ }^{1}$ and Ari Waisman ${ }^{2}$
}

\begin{abstract}
${ }^{1}$ Neuroimmunology Division, Institute of Experimental Immunology, Department of Pathology, University Hospital Zurich, Zurich, Switzerland ${ }^{2}$ First Medical Department, University of Mainz, Mainz, Germany. ${ }^{3}$ Department of Neuropathology, Charité — Universitätsmedizin Berlin,
\end{abstract} Berlin, Germany. ${ }^{4}$ Merck Serono International, Geneva, Switzerland.

\begin{abstract}
The clear association of Th17 cells with autoimmune pathogenicity implicates Th17 cytokines as critical mediators of chronic autoimmune diseases such as EAE. To study the impact of IL-17A on CNS inflammation, we generated transgenic mice in which high levels of expression of IL-17A could be initiated after Cre-mediated recombination. Although ubiquitous overexpression of IL-17A led to skin inflammation and granulocytosis, T cell-specific IL-17A overexpression did not have a perceptible impact on the development and health of the mice. In the context of EAE, neither the T cell-driven overexpression of IL-17A nor its complete loss had a major impact on the development of clinical disease. Since IL-17F may be able to compensate for the loss of IL-17A, we also generated IL-17F-deficient mice. This strain was fully susceptible to EAE and displayed unaltered emergence and expansion of autoreactive $T$ cells during disease. To eliminate potential compensatory effects of either cytokine, we treated IL-17F-deficient mice with antagonistic monoclonal antibodies specific for IL-17A and found again only a minimal beneficial impact on disease development. We conclude therefore that both IL-17A and IL-17F, while prominently expressed by an encephalitogenic $T$ cell population, may only marginally contribute to the development of autoimmune CNS disease.
\end{abstract}

\section{Introduction}

MS and its animal model EAE are characterized by the invasion of self-reactive Th cells into the CNS, leading to demyelination, axonal loss, and neurological impairment (1). Upon activation, Th cells can differentiate into different effector cells, depending on the makeup of the priming immune synapse as well as the cytokines present (2). For over a decade, IFN- $\gamma$-secreting Th1 cells were thought to be the pathogenic population central to the pathogenesis of autoimmunity, considering the clear association of the Th1 effector type to diseases like rheumatoid arthritis, MS, and type 1 diabetes. In their respective animal models, however, the loss of the major Th1 cytokines, IFN- $\gamma$, IL-12, and IL-18 surprisingly did not hamper disease development (3-5). In fact, IFN- $\gamma$ and IL-12 deficiency led to, clinically, even more severe inflammation in collagen-induced arthritis (CIA) and $\operatorname{EAE}(3,5,6)$. After these discoveries the simplistic notion that Th1 cells and their respective cytokines are the culprits of autoimmunity had to be revised. In contrast to IL-12, its relative IL-23 was found to be essential for the development of EAE and CIA (7). The finding that IL-23 induces the expression of IL-17A by Th cells then gave rise to the notion that not Th1 but IL-17A-secreting Th cells (Th17) are the main pathogenic population in autoimmune diseases (8). This hypothesis was strongly supported by adoptive transfer models, in which the transfer of IL-17A-producing effector cells into WT hosts resulted in the initiation of autoimmunity $(8,9)$. The de novo lineage commitment of naive T cells toward IL-17A secretion is dependent on TGF- $\beta$ R engagement (10). Additional IL-6 signaling has been identified as

Authorship note: Stefan Haak and Andrew L. Croxford contributed equally to this work. Burkhard Becher and Ari Waisman contributed equally to this work.

Conflict of interest: The authors have declared that no conflict of interest exists. Nonstandard abbreviations used: MOG, myelin oligodendrocyte glycoprotein. Citation for this article: J. Clin. Invest. doi:10.1172/JCI35997. a costimulus, directing cell fate toward Th17 commitment. IL-17A secretion is considered the hallmark of Th17 function, as it exhibits strong proinflammatory properties $(8,11)$ and is widely held as being the major driving force in the pathogenesis of autoimmunity. Despite the plethora of data published on IL-17A implicating its function in physiological processes, hardly any studies reveal a true causative association (reviewed in ref. 12). Here we have generated mice in which $\mathrm{T}$ cells overexpress IL-17A and found that even strongly increased delivery of IL-17A by $\mathrm{T}$ cells into in the inflamed CNS has no impact on the pathogenesis of EAE. Also, we confirmed that $I l 17 a^{-/}$mice are fully susceptible to EAE. It is feasible that other Th17 cytokines can compensate for the loss of IL-17A. The closest associate to IL-17A is $\operatorname{IL}-17 F(13,14)$, which is encoded in a syntenic fashion and shares around $50 \%$ sequence homology and a strikingly similar pattern of expression with IL-17A (15). IL-17A/F heterodimers were described previously (16) and have been shown to signal through the same receptor complex $(17,18)$. In order to determine whether IL-17F contributes to the pathogenicity of Th17 cells, we generated $I l 17 f^{\wedge}-$ mice and explored their susceptibility to EAE. To prevent potential compensatory effects of IL-17A in $I l 17 f^{-/}$mice, we treated them with antagonistic anti-IL-17A mAbs. Surprisingly, we discovered even anti-IL-17Atreated $I l 17 f /$ mice to be fully susceptible to EAE, indicating that while Th17 cells physiologically associate with an encephalitogenic state, neither IL-17A nor IL-17F appear to contribute substantially to the pathogenic function of Th17 cells in vivo.

\section{Results}

IL-17A-overexpressing $T$ cells do not enhance the pathogenesis and clinical development of myelin oligodendrocyte glycoprotein-induced EAE. To address the impact of IL-17A expressed by CNS-invading $\mathrm{T}$ cells on the pathogenesis of EAE, we generated a mouse conditionally overexpressing IL-17A together with EGFP (termed 
A CD4-IL17A ind/ $/+^{-}$
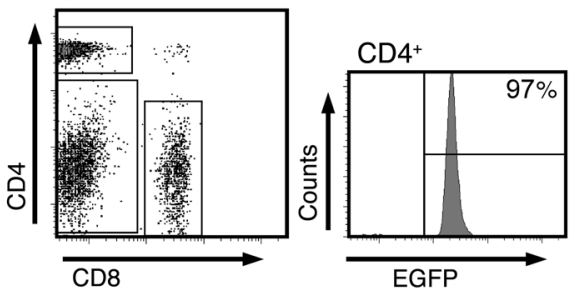

CD8

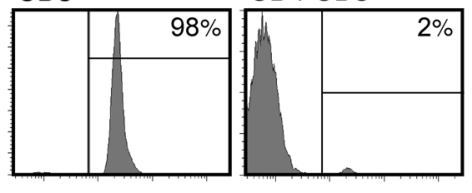

IL-17A $A^{\text {ind } /+}$
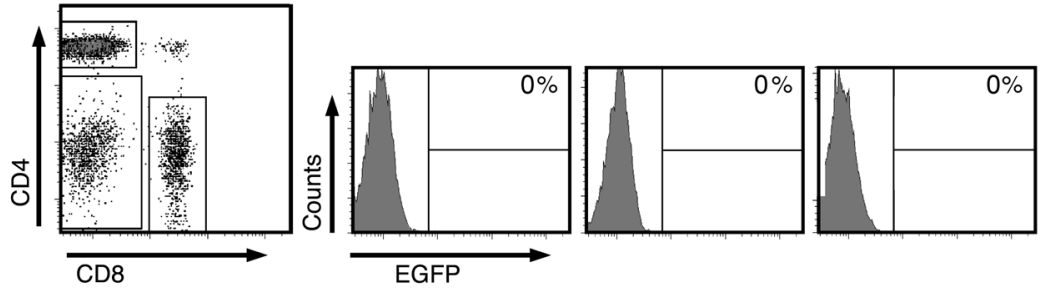

C
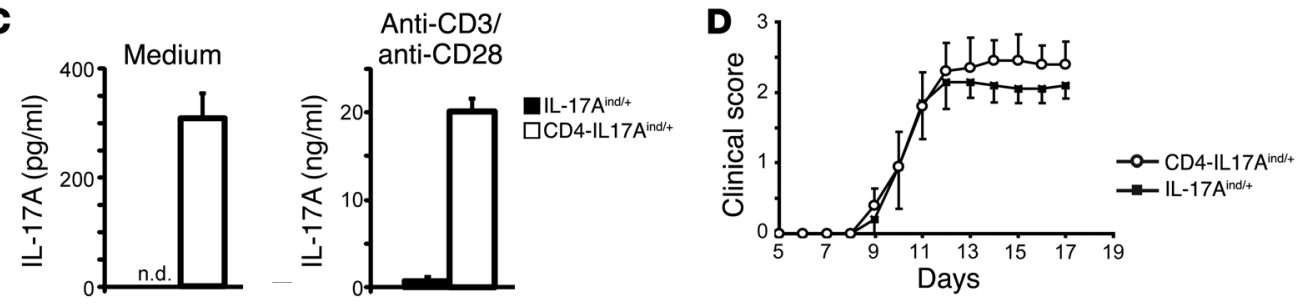

E
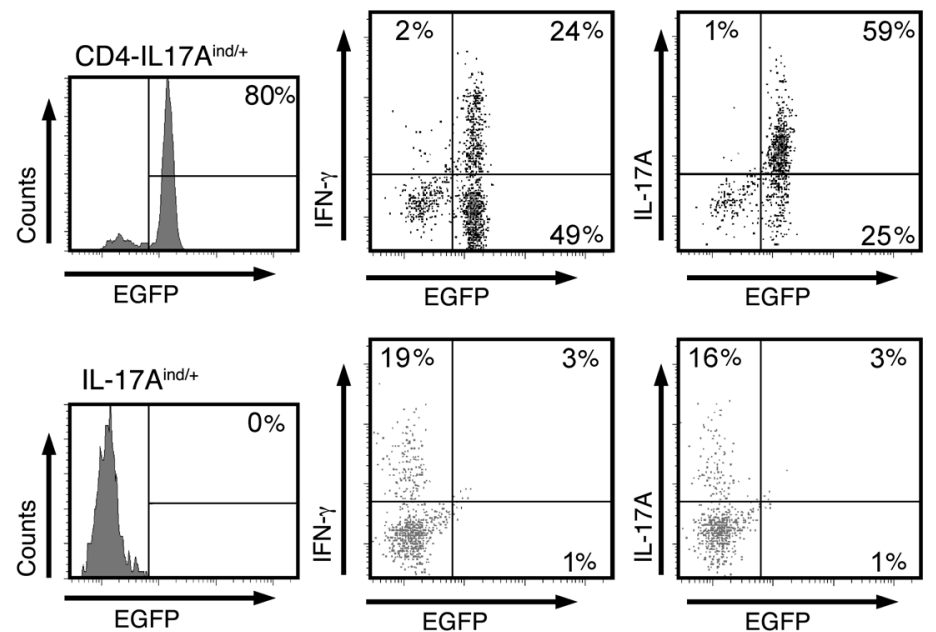

B EGFP-
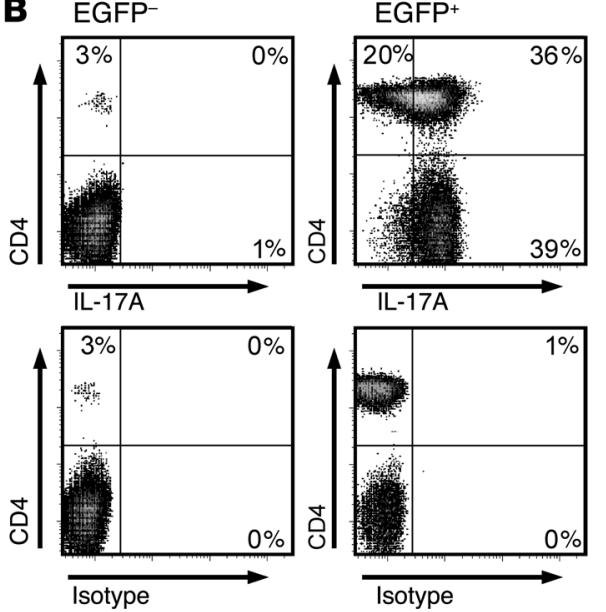

$\mathbf{F}$
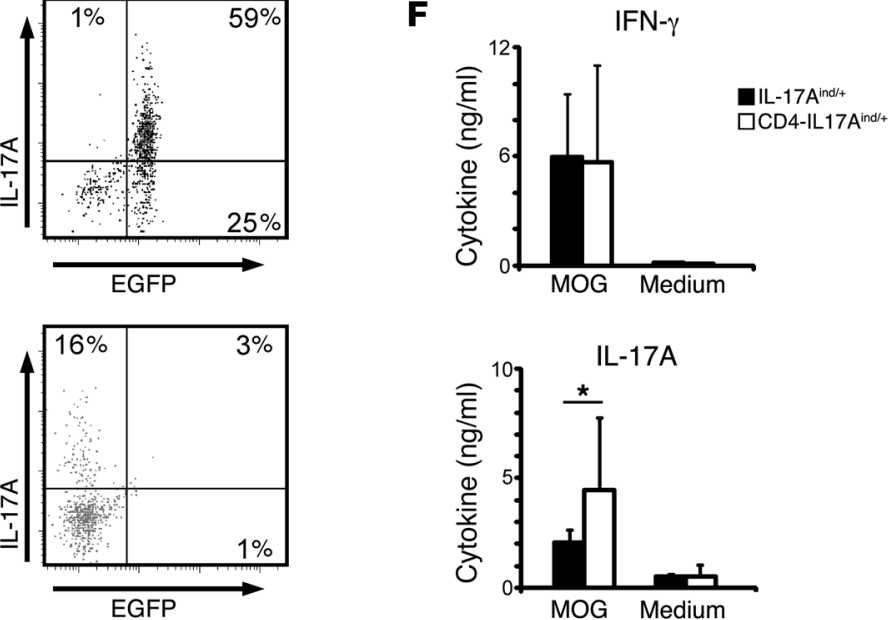

IL-17A

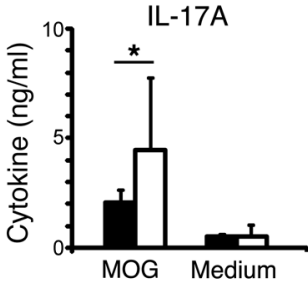

Figure 1

IL-17A overexpression does not exacerbate EAE. (A) LNs from CD4-IL17A ind/+ and IL-17A ind/+ littermates were isolated and stained for CD4 and CD8 coreceptors. EGFP expression is depicted in the histograms after indicated gating. Percentages of gated cells are shown. (B) Splenocytes from naive CD4-IL17A ind/+ mice were restimulated in the presence of Brefeldin A and subsequently stained for CD4 and IL-17A. Percentages of cells in the quadrants are indicated in the corners after gating was performed on all EGFP- (plots on the left) or all EGFP+ (plots on the right) cells. Data shown are representative of 3 independent experiments. (C) FACS-sorted CD4 $4^{+}$cells $\left(2 \times 10^{5}\right)$ were cultured for 24 hours in the presence or absence of anti-CD3 and anti-CD28, after which IL-17A secretion was measured by flow cytomix assay. Error bars represent mean \pm SD. n.d., not detectable. (D) Clinical scores after $M O_{35-55}$-induced EAE are not significantly altered by increased IL-17A expression in CD4-IL17A ind/+ and IL-17A ind/+ littermates. Error bars represent mean \pm SEM. Data shown represent 1 out of 3 independent experiments.

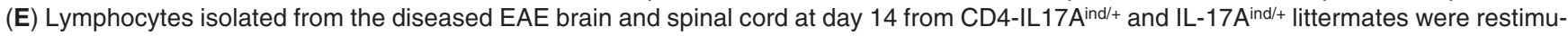
lated and surface stained for CD4 and examined for EGFP expression. Further staining for IL-17A and IFN- $\gamma$ was performed. Percentages of EGFP+IL-17A+ or EGFP+IFN- $\gamma^{+}$are given in the quadrant corners. Plots shown are gated on CD4+ CNS-derived T cells. (F) At peak disease,

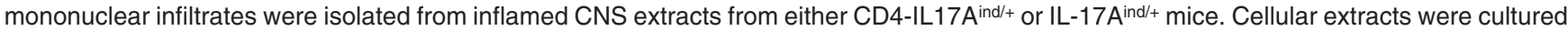
for 2 days in the presence of $20 \mu \mathrm{g} / \mathrm{ml}$ MOG peptide, after which cytokine secretions were measured by flow cytomix. Error bars represent mean $\pm \mathrm{SD}$ and significance is shown where relevant. ${ }^{*} P=0.039$, Student's $t$ test. 


\section{Table 1}

Detailed clinical development of EAE in IL-17A-overexpressing, IL-17A-, and IL-17F-deficient mice

\begin{tabular}{|c|c|c|c|}
\hline & $\begin{array}{l}\text { Day of } \\
\text { onset }^{A}\end{array}$ & Incidence & $\begin{array}{l}\text { Maximum } \\
\text { score }^{A}\end{array}$ \\
\hline $\begin{array}{l}\text { CD4-IL17Aind/+ } \\
\text { IL-17A }\end{array}$ & $\begin{array}{l}10 \pm 0.5 \\
10 \pm 0.3\end{array}$ & $\begin{array}{l}93 \%(13 / 14) \\
92 \%(12 / 13)\end{array}$ & $\begin{array}{l}2.91 \pm 0.29 \\
2.67 \pm 0.13\end{array}$ \\
\hline $\begin{array}{l}1 / 17 a^{-/-} \\
1117 a^{+/+}\end{array}$ & $\begin{array}{r}10 \pm 0.3 \\
9 \pm 0.4\end{array}$ & $\begin{array}{r}91 \%(32 / 35) \\
100 \%(46 / 46)\end{array}$ & $\begin{array}{l}2.36 \pm 0.13 \\
2.75 \pm 0.19\end{array}$ \\
\hline $\begin{array}{l}1 / 17 f^{-1-} \\
I 117 f^{+--} \\
I 117 f^{+/+}\end{array}$ & $\begin{array}{l}11 \pm 0.3 \\
10 \pm 0.3 \\
12 \pm 0.7\end{array}$ & $\begin{array}{r}95 \%(37 / 39) \\
90 \%(38 / 42) \\
100 \%(17 / 17)\end{array}$ & $\begin{array}{l}2.68 \pm 0.10 \\
2.74 \pm 0.12 \\
2.67 \pm 0.15\end{array}$ \\
\hline $\begin{array}{l}1 / 17 f^{-/-}+\alpha I \mathrm{IL}-17 \mathrm{~A} \\
\| 117 f^{--}+\text {Iso. ctrl. }\end{array}$ & $\begin{array}{l}15 \pm 0.8 \\
13 \pm 0.5\end{array}$ & $\begin{array}{l}75 \%(9 / 12) \\
83 \%(10 / 12)\end{array}$ & $\begin{array}{l}2.47 \pm 0.29 \\
2.70 \pm 0.19\end{array}$ \\
\hline
\end{tabular}

AMean of diseased mice (Mean \pm SEM). Iso. ctrl., isotype control.

IL-17 ${ }^{\text {ind }}$ ) after excision of a loxP-flanked transcriptional STOP cassette (Supplemental Figure 1A). LN cells from IL-17A ${ }^{\text {ind } /+}$ mice were shown to functionally express both EGFP and secrete highly upregulated levels IL-17A after in vitro Cre-mediated recombination (Supplemental Figure 1, B and C). Crossing the IL-17A $\mathrm{A}^{\text {ind }}$ with CD4-Cre-expressing mice (termed CD4-IL17A ${ }^{\text {ind }}$ ) generated a $\mathrm{T}$ cell repertoire, in which both $\mathrm{CD}^{+}$and $\mathrm{CD}^{+} \mathrm{T}$ cells excise the STOP cassette and express the EGFP reporter (Figure 1A). CD4IL17 $\mathrm{A}^{\text {ind/+ }} \mathrm{T}$ cells constitutively produce IL-17A in the steady state, and this is shown to directly correlate with expression of EGFP (Figure 1B). Expression of IL-17A by T cells in CD4-IL17A ${ }^{\text {ind/++ }}$ mice did not result in an altered composition of thymus or spleen (Supplemental Table 1). Considering that IL-17A has been shown to affect granulopoiesis (19), we examined granulocyte homeostasis in the CD4-IL17A ${ }^{\text {ind }}$ strain. In naive mice, we observed a minor but significant increase in the number of granulocytes in peripheral blood (Supplemental Figure 1D). After immunization of CD4-IL17 $\mathrm{A}^{\text {ind/+ }}$ and littermate controls with myelin oligodendrocyte glycoprotein 33-55/CFA (MOG $35-55 / \mathrm{CFA})$, we observed a highly significant increase in serum IL-17A, which correlated to enhanced neutrophil recruitment into the spleen (Supplemental Figure 1, E and F), thus confirming in vivo the ectopic activity of IL-17A produced by CD4-IL17A $\mathrm{A}^{\text {ind/+ }} \mathrm{T}$ cells.

To verify the increased IL-17A secretion in CD4-IL17A ${ }^{\text {ind/+ }} \mathrm{T}$ cells, we performed IL-17A-specific ELISA assays with FACS-sorted CD4-IL17A ${ }^{\text {ind/+ }}$ and IL-17A ${ }^{\text {ind/+ }}$ CD4 $4^{+} \mathrm{T}$ cells after 24 hours in the presence or absence of CD3 and CD28 cross-linking. As expected, CD4-IL17A $\mathrm{A}^{\text {ind/+ }} \mathrm{CD} 4^{+} \mathrm{T}$ cells constitutively secreted elevated levels of IL-17A compared with control IL17A ${ }^{\text {ind/+ }} \mathrm{T}$ cells. This secretion was greatly enhanced after polyclonal stimulation with antiCD3/anti-CD28 (Figure 1C). Expression of other Th-associated cytokines such as IFN- $\gamma$, IL-4, and IL-2 remained indistinguishable from control $\mathrm{T}$ cells in both stimulated and unstimulated cultures (data not shown). Next, we immunized CD4-IL17A ${ }^{\text {ind/+ }}$ mice with $\mathrm{MOG}_{35-55} / \mathrm{CFA}$ and pertussis toxin to induce and follow progression of EAE. Surprisingly, no significant clinical differences were observed between CD4-IL17 $\mathrm{A}^{\text {ind/+ }}$ and IL-17A $\mathrm{A}^{\text {ind } /+}$ littermates in a series of experiments (Figure 1D and Table 1). Consistent with the clinical disease, cellular CNS invasion was virtually identical in both groups as measured by flow cytometry (data not shown). From all CNS fractions analyzed, no significant alterations were observed with respect to IFN- $\gamma$-secreting cells. However, CNS extracts from CD4-IL17 ${ }^{\text {ind/+ }}$ mice presented with a clearly identifiable and consistent increase in IL-17A ${ }^{+} \mathrm{T}$ cells compared with IL-17A $\mathrm{A}^{\text {ind/+ }}$ mice (Figure 1E). Despite similar clinical scores, flow cytometric cytokine analysis revealed a significant increase in IL-17A secretion from CNS-isolated CD4-IL17A ${ }^{\text {ind/+ }} \mathrm{T}$ cells, while other proinflammatory cytokines associated with EAE, including IFN- $\gamma$ (Figure 1F), IL-6 or GM-CSF (data not shown), remained unaltered. Taken together, exacerbated $\mathrm{T}$ cell-mediated delivery of IL-17A into the inflamed CNS during MOG-induced EAE does not result in an appreciable alteration of the course of disease.

IL-17A $\mathrm{A}^{\text {ind/+ }}$ mice were also crossed with the deleter-cre strain (20). These mice (termed Del-IL17A ${ }^{\text {ind }}$ ) showed early signs of skin inflammation and, ultimately, a developmental retardation clearly visible from P4-6 on, as can be seen in Figure 2A. This phenotype was coupled with an upregulated secretion of IL-17A by cells isolated from bone marrow, spleen, thymus, and mesenteric LNs in unstimulated cultures (Figure 2B). The IL-17A overexpression also led to a substantial increase in the number of granulocytes throughout the body, especially in bone marrow, spleen, and blood (Figure 2, C and D). Chemical analysis of the blood compartment revealed an anemia-like phenotype, consistent with granulocytosis (Table 2). We are in the process of further elucidating the clinical impact of this genotype (unpublished observations).

$I L-17 A$ function is redundant in the development of EAE. In order to improve the understanding of the role of the Th17 effector type in autoimmunity, we next analyzed the impact of loss of IL-17A on the autoreactive Th17 response in EAE. Despite the close association of IL-17A with the inflammatory milieu in EAE, we confirmed that the loss of IL-17A does not fundamentally impede the induction of the disease, which is similar to the observations made by Iwakura and colleagues (21) (Figure 3A). In the course of EAE, there was an apparent mild decrease of disease severity. Overall, the disease severity in IL-17A-deficient mice was significantly decreased (analysis of covariance, $P=0.015$ ). However, the difference in the course of the disease between WT and IL-17A-deficient mice accounted for only $1.6 \%$ of the variance in the experiment, which shows how small the effect size is compared with the variance in the system (e.g., the "mouse identity" [as in residual intermouse variance] accounts for $24 \%$ of the variance). In a detailed analysis of the pooled data, we observed only a minimal, yet statistically significant (2-tailed $t$ test, $P=0.013$ ), difference in the day of onset of disease and an insignificant decrease in incidence and the maximal severity of disease (Table 1). The nonessential role of IL-17A in EAE development may be due to the involvement of other Th17 associated factors. To elucidate the quality of the Th17 response in the immunized IL-17A-deficient mice, we analyzed their cytokine secretion upon in vitro restimulation with MOG $_{35-55}$ peptide, either in the absence of exogenous cytokines or under Th17 polarizing conditions (Figure 3, B and C). Surprisingly, while the IL-22 levels were similar with T cells obtained from IL-17A-deficient and WT control mice, IL-17F secretion was found to be consistently elevated in the IL-17A-deficient model. This specific increase of 1 Th17 cytokine suggests the possibility of a compensatory expression of IL-17F in the absence of IL-17A, which may contribute to disease development.

IL-17F is expressed by Th17 cells and is abundant in the inflamed CNS. A screen performed by quantitative RT-PCR of cerebelli of mice with active EAE revealed that $I l 17 f$, like $I l 17 a$, was highly expressed in the lesioned CNS as compared with cerebelli of healthy con- 


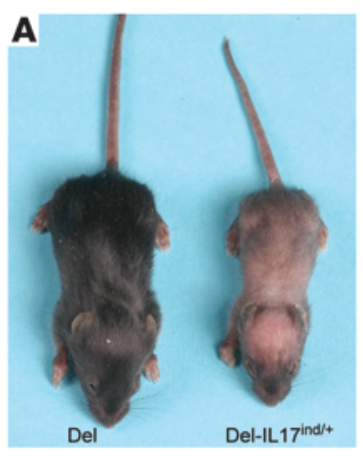

B
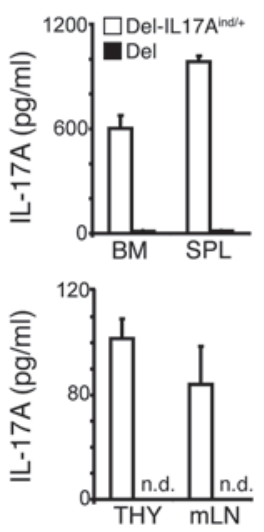
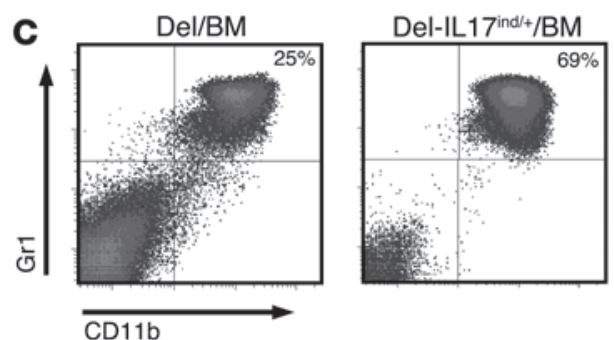

D
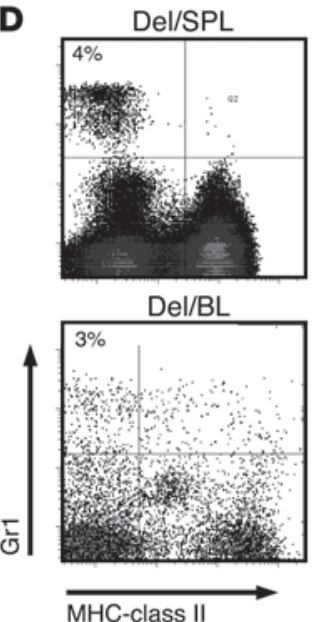

Del-IL17 ${ }^{\text {ind } /+} /$ SPL

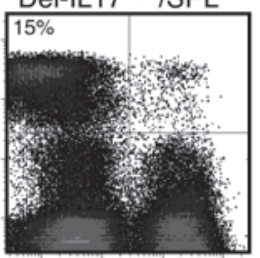

Del-IL17 ${ }^{\text {ind } / 4 / B L}$

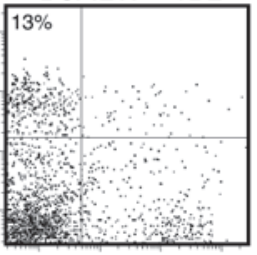

\section{Figure 2}

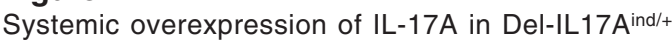
leads to granulocytosis and anemia. (A) Crossing the IL-17A ind allele to the delete-cre strain yields mice called Del-IL17A ind/+. Skin inflammation, stunted growth, and failure to thrive is a consistent phenotype in all mice observed when compared with delete-cre littermate controls (Del). (B) Single-cell suspensions from bone marrow, spleen (SPL), thymus (THY), and mesenteric LNs $(\mathrm{mLN})$ were placed in unstimulated culture for 24 hours, after which IL-17A secretion was assayed by ELISA. Error bars represent mean \pm SEM. (C) Bone marrow cells were isolated from Del-IL17A $A^{\text {ind/+ }}$ mice and littermate controls and surface stained for Gr1 and CD11b. Percentages of gated granulocytes are shown in quadrant corners. (D) Isolated spleen cells and PBMCs from the indicated genotypes were stained for MHC-class II and Gr1. Percentages of gated cells are shown. (A-D) Data shown are representative of at least 2 independent experiments. trols (Figure 3D). To assess the source of IL-17F in the context of EAE, we restimulated in vivo primed splenocytes in vitro with MOG $_{35-55}$ under Th17 polarizing conditions and subsequently analyzed the cytokine profile by intracellular cytofluorometric analysis. IL-17F expression was restricted to MOG-responsive and potentially encephalitogenic $\mathrm{CD}^{+}{ }^{+} \mathrm{Th}$ cells that are also producing IL-17A (Figure 3E).

The observation that both cytokines are found in the inflamed CNS and mark those highly pathogenic Th17 cells that have been associated with autoreactive lesions suggests that IL-17F is an encephalitogenic cytokine with functional relevance in CNS autoimmune inflammation.

Generation and analysis of the $I L-17 F-$ deficient mice. To ultimately determine whether IL-17F contributes to the development of EAE, we generated IL-17F-deficient mice by the replacement of exons 2 and 3 with a lacZ reporter cassette. IL-17F deficiency was confirmed by ELISA (Supplemental Figure 1G). Homozygous offspring were viable and showed neither developmental malformation nor any evident immunodeficiency under SPF conditions. There were no apparent alterations in the cellular composition of the immune system in homeostasis as shown by FACS analysis for spleen and thymus in Supplemental Table 2.

Up to 42 mice per group $(+/+,+/-,-/-)$ in 6 independent experiments were immunized with $\mathrm{MOG}_{35-55} / \mathrm{CFA}$, and the clinical development of EAE was monitored daily. In line with the report by Dong and colleagues (22), the lack of IL-17F did not have any visible consequences on clinical EAE development as shown in Figure 4A. Il17f ${ }^{-1-}$ mice did not show any alteration in the day of disease onset, maximum score, or incidence when compared with IL-17F competent $(+/+,+/-)$ mice (Table 1$)$. In accordance

\section{Table 2}

Chemical analysis of the blood compartment

\begin{tabular}{|c|c|c|c|c|c|c|}
\hline Blood & \multicolumn{3}{|c|}{ Del-IL17A $A^{\text {ind } /+A}$} & \multicolumn{3}{|c|}{ DelB } \\
\hline wbc $\left(\times 10^{3}\right.$ cells $\left./ \mu \mathrm{l}\right)$ & 4.20 & 1.77 & 1.35 & 1.53 & 1.50 & 3.63 \\
\hline $\mathrm{rbc}\left(\times 10^{6} \mathrm{cells} / \mu \mathrm{l}\right)$ & 6.18 & 5.34 & 3.45 & 4.36 & 8.74 & 7.98 \\
\hline Hemoglobin $(\mathrm{g} / \mathrm{dl})^{\mathrm{C}}$ & 9.00 & 7.80 & 8.00 & 14.20 & 14.60 & 12.60 \\
\hline Hematocrit $(\%)^{D}$ & 31.20 & 27.30 & 29.50 & 46.00 & 47.20 & 44.80 \\
\hline Mean corpuscular volume (fl) ${ }^{\mathrm{E}}$ & 50.40 & 50.90 & 46.10 & 53.90 & 54.00 & 56.00 \\
\hline Mean corpuscular hemoglobin $(p g)^{\mathrm{F}}$ & 14.50 & 14.60 & 12.50 & 16.20 & 16.70 & 15.7 \\
\hline Mean corpuscular hemoglobin concentration $(\mathrm{g} / \mathrm{dl})$ & 28.70 & 28.70 & 27.00 & 30.00 & 30.90 & 28.00 \\
\hline
\end{tabular}

${ }^{\mathrm{A}} \mathrm{IL}-17 \mathrm{~A}^{\mathrm{ind} /+}$ mice crossed with the deleter-cre strain. ${ }^{\mathrm{B}}$ deleter-cre control littermates. ${ }^{\mathrm{C} P}=0.0015 .{ }^{\mathrm{D}} P=0.0002 .{ }^{\mathrm{E}} P=0.0301 .{ }^{\mathrm{F}} P=0.0347 . \mathrm{Routine} \mathrm{CBC}$ analysis was performed on blood samples from the indicated mice $(n=3)$. Each column represents an individual mouse. Significant alterations in values are shown. Data shown are representative of at least 2 independent experiments. 
A

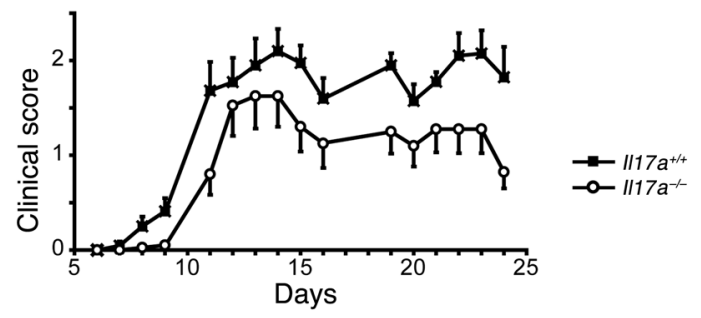

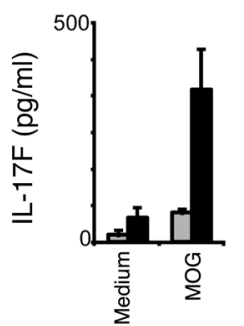

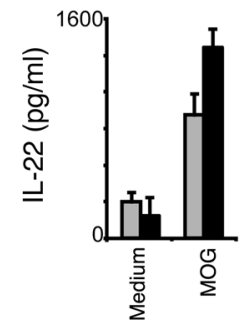

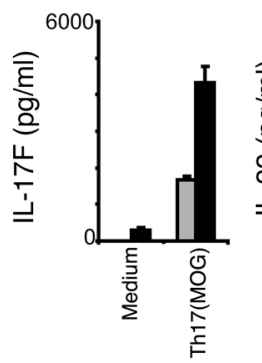

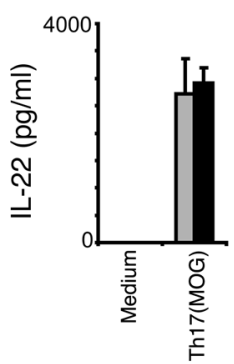

D
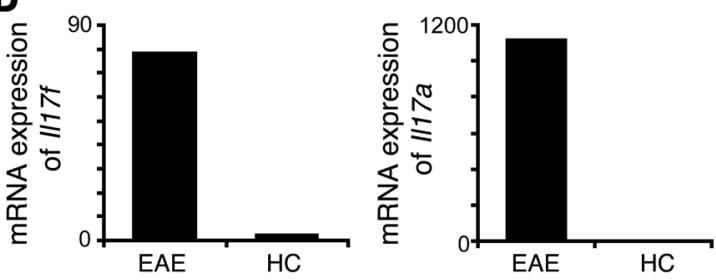

E
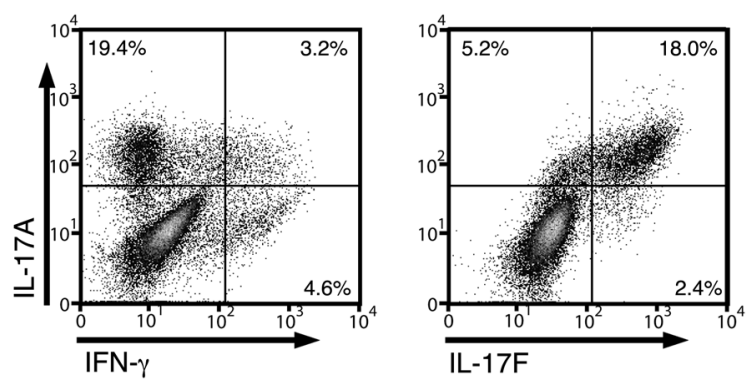

with clinical EAE scores, histological analysis of spinal cord cross sections displayed no discernable features among WT mice and heterozygous $I l 17 f^{+/-}$and homozygous $I l 17 f^{/-}$mice. Inflammation caused a severe impairment of myelinated and axonal structures, subsequently inducing reactive astrogliosis (Figure 4B). Detailed analysis of CNS-infiltrating cells by flow cytometry revealed no change in cell numbers or makeup of CNS-invading leukocytes (Figure 4C).

We did not observe any relevant difference in the capacity of $I l 17 f^{/-}$mice to initiate $\mathrm{CD}^{+} \mathrm{T}$ cell priming and the effector $\mathrm{T}$ cell response. Il17f ${ }^{-}$- and control mice were immunized against $\mathrm{MOG}_{35-55}$, and primed lymphocytes were isolated from draining LNs prior to disease onset (Figure 5, A-C) and were subsequently

\section{Figure 3}

$\mathrm{IL}-17 \mathrm{~A}$ is redundant in the induction of EAE, which could be due to a compensatory increase of IL-17F production. (A) EAE was induced in $/ 117 a^{-/-}$and $/ 117 a^{+/+}$mice by immunization with $\mathrm{MOG}_{35-55} / \mathrm{CFA}$. The graph shows the development of EAE according to clinical scores ( $n=10$; SEM as indicated) in 1 out of 2 independent experiments. (B and C) Th17-cytokine profile measured by ELISA of splenocytes isolated from mice with active EAE and restimulated with $\mathrm{MOG}_{35-55}$ with (C) or without (B) the addition of Th17 polarizing conditions for 2 days. Error bars represent mean \pm SEM. (D) Comparative mRNA expression analysis of $I / 17 f$ and $I / 17 a$ in the cerebellum of mice at peak EAE versus healthy controls $(\mathrm{HC})$. The data represent 1 of 2 independent experiments $(n=4)$. (E) Th17 cells were generated in vitro from $\mathrm{MOG}_{35-55}$-immunized $\mathrm{C} 57 \mathrm{BL} / 6$ mice. Splenocytes were harvested 7 days after immunization, Th17 polarized, and analyzed by intracellular cytokine staining for IL-17A, IL-17F, and IFN- $\gamma$. Percentages of gated cells are shown. A representative of 3 independent experiments is shown.

challenged in vitro with their cognate antigen. Lymphocytes from all groups responded with the same degree of proliferation (Figure 5C). The full susceptibility of $I l 17 f^{-} /$mice to EAE could have resulted from a compensatory increase in the production of IL-17A. However, Il17f/- lymphocytes obtained from the spleen showed a consistent decrease in the production of IL-17A (Figure 5A) and the frequency (Figure 5B) of IL-17A-secreting cells. Even upon overwhelming in vitro polarization toward the Th17 lineage, the proportion of IL-17A-producing T cells and the overall amount of secreted IL-17A decreased in IL-17F-targeted cells compared with WT cells under our in vitro conditions (Figure 5D). While our data indicate some degree of haploinsufficiency of the IL-17F allele (Figure 5E and Supplemental Figure 1H), targeting this locus had an inhibitory impact on IL-17A expression in vitro. We next addressed whether CNS-invading Th17 cells of the Il17f $/$ mice express altered levels of IL-17A, but, in contrast to the in vitro results, we found no significant difference (Figure 5F).

Neutralization of IL-17A in IL-17F-deficient mice does not diminish $E A E$. Given the shared receptor specificities of IL-17F in IL-17A, the possibility exists that one cytokine is able to compensate for the loss of the other in vivo. Therefore, without removing both cytokines from EAE disease progression, redundancy of either IL-17A or IL-17F is open to interpretation. To remove both IL-17A and IL-17F during the course of EAE, we treated IL-17F-deficient mice twice a week with $200 \mu \mathrm{g}$ of an antagonistic anti-IL-17A mAb (9). Upon repeated treatment of WT and $I l 17 f^{-/}$mice with either anti-IL-17A or rat-IgG1 isotype control, we could verify the titer (approximately $125 \mu \mathrm{g} / \mathrm{ml}$ ) of the agonist in peripheral blood. The blocking capacity of the antibody found in those mice compared with the neutralizing antibody titrated directly on an IL-17A protein standard (Supplemental Figure 2A). To further control for the capacity of anti-IL-17A mAbs to block IL-17A, we treated immunized CD4-IL17 $7^{\text {ind/ }}$ mice with $100 \mu \mathrm{g}$ of anti-IL-17A and found that even the accumulation of serum IL-17A in the overexpressing mice could be completely abolished (Supplemental Figure 2B). Thus, our in vivo neutralization protocol was confirmed to be an appropriate method to induce IL-17A deficiency in our mouse models. To implement this system in our clinical investigations, we treated $I l 17 f^{-/}$mice with anti-IL-17A mAbs. We discovered a slight trend toward disease amelioration (Figure 6), which matches our findings using $\mathrm{Il} 17 \mathrm{a}^{-/}$mice and the observations by Hofstetter et al. (23). While blockade of IL-17A has some beneficial effect, we 

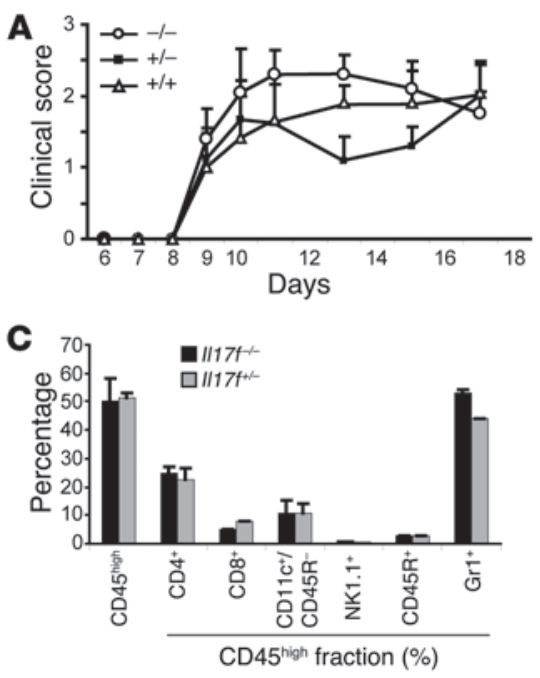

B

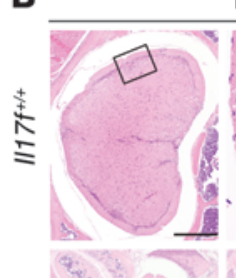

$H \& E$

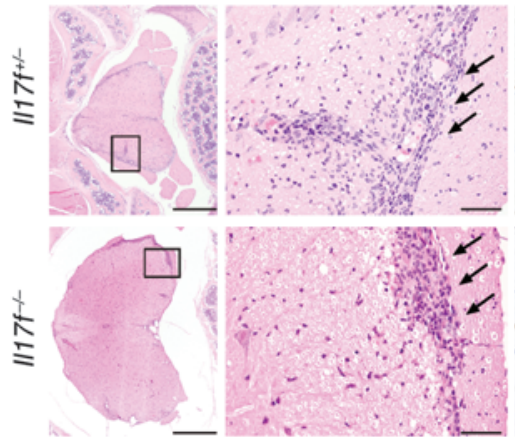

GFAP

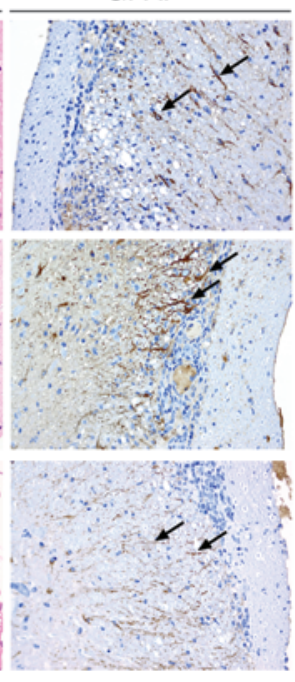

CNPase

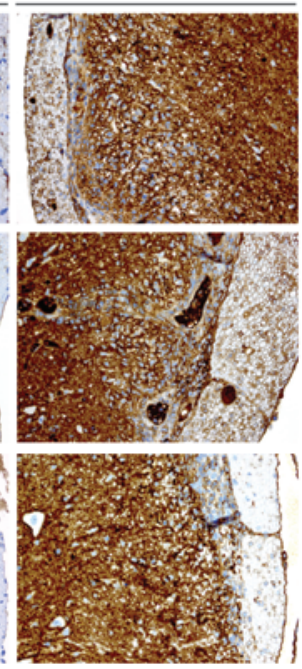

\section{Figure 4}

IL-17F is not required for the development of EAE. (A) EAE was induced in $/ / 17 f^{-/-}$, $/ / 17 f^{-/+}$, and $/ / 17 f^{+/+}$mice by immunization with $\mathrm{MOG}_{35-55} / \mathrm{CFA}$. The graph shows the development of EAE according to clinical scores in 1 out of 5 independent experiments. Error bars represent mean \pm SEM. (B) Spinal cord cross sections, in accordance with clinical EAE scores, displayed similar inflammatory lesions (H\&E staining; arrows). Inflammation caused an impairment of myelinated structures (staining for CNPase) and induced a reactive astrogliosis (staining for glial fibrillary acidic protein [GFAP]). All stainings were performed on serial sections. Scale bars: $500 \mu \mathrm{m}$ (first column); $50 \mu \mathrm{m}$ (second, third, and fourth columns). Rectangle in first column represents the area shown in second, third, and fourth columns. (C) Detailed analysis of infiltrating lymphocytes into cerebellum and spinal cord was performed by cytofluorometric analysis of surface marker staining. CD45high cells represent the CNS-invading leukocytes, which were gated on for detailed analysis. Error bars represent mean \pm SEM.

conclude that IL-17A and IL-17F, alone or in tandem, do not contribute critically to the development or progression of EAE.

\section{Discussion}

After the discovery of distinct $\mathrm{T}$ cell polarization patterns by Mosmann and colleagues (24), it was accepted for more than a decade that IFN- $\gamma$-secreting Th 1 cells are the main encephalitogenic population in CNS inflammatory diseases such as MS and EAE. In addition, Th1-promoting factors such as IL-12 and IL-18 were considered indispensable for the initiation of autoimmune disease in mice. This simplistic paradigm had to be revised when it was discovered that mice deficient in IFN- $\gamma$, TNF- $\alpha$, IL-12p35, and IL-18 are either fully susceptible to EAE or hypersusceptible (3-5, 25 ). While IL-12 is dispensable for the induction of EAE, its close relative IL-23 has been demonstrated to be absolutely essential. Langrish et al. initially were able to show that IL-23 induces the secretion of IL-17A by effector T cells (9). IL-17A-secreting effector $\mathrm{T}$ cells were ultimately termed Th17 cells and are now established as a distinct helper $T$ cell subset. In addition, the close association of Th17 cells with inflammatory autoimmune diseases such as rheumatoid arthritis, MS, and psoriasis has clearly marked this population as pathogenic (8). Several reports have elucidated the conditions to polarize toward this lineage in vitro $(8,10)$. While IL-17A is now considered to be the main driving force behind tissue inflammation, to this day, virtually all claims are based on a correlative relationship between Th17 cells and their presence in an inflammatory lesion. Assuming that IL-17A drives the inflammatory process and could contribute to blood brain barrier breakdown (26) and increased neutrophil activity (19), we generated transgenic mice, in which T cells produce high levels of IL-17A.
Surprisingly, however, greatly increased levels of $\mathrm{T}$ cell-derived IL-17A expression did not impact on the development of EAE or on the quality and quantity of inflammation in the CNS. Hofstetter et al. were the first to block IL-17A in EAE and found only a minimal efficacious effect in vivo, regardless of whether they blocked IL-17A alone with an antagonistic mAb or IL-17A and IL-17F combined using a soluble IL-17 receptor (23). Komiyama et al. previously addressed the role of IL-17A in EAE by generating a deficient mouse strain (21). Unlike the deficiencies in IL-23 and IL-6, which render mice completely resistant to EAE, loss of IL-17A does not prevent disease development. However, IL-17A-deficient mice display a significantly less severe disease at late time points (26 days after immunization) and a major loss of encephalitogenic capacity after adoptive transfer of in vitro expanded $T$ cells. However, the cells were kept in culture for 4 days and the impact on Th polarization was not evaluated. Therapeutic targeting of IL-17 using a vaccination strategy showed a more robust efficacy but did not lead to complete resistance to EAE (27). The far more critical role of IL-23 and IL- 6 in the development of autoimmune inflammation indicates that the associated disruption of IL-17A and IL-17F production is a symptom but not the main cause of the complete EAE resistance observed in IL-23- and IL-6-deficient mice. Matching our conclusions, in other models of autoimmunity such as experimental autoimmune uveitis (EAU), the function of IL-17A appears to be redundant (28). Luger et al. recently demonstrated that while IL-17 can participate in the pathogenesis of EAU, it has by no means an essential role (29). The concept that IL-17A itself is not likely the only pathogenic molecule generated by Th17 cells was further supported by the report of McGeachy et al., who could demonstrate that IL-23-driven Th cells but not TGF- $\beta$ / 

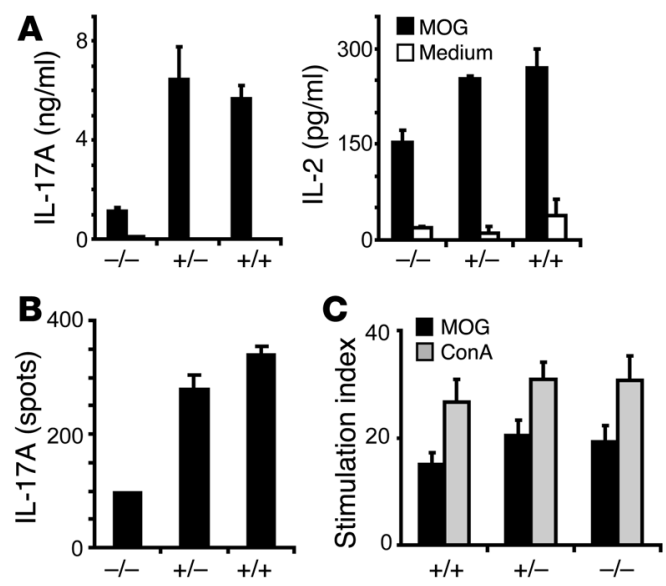

D
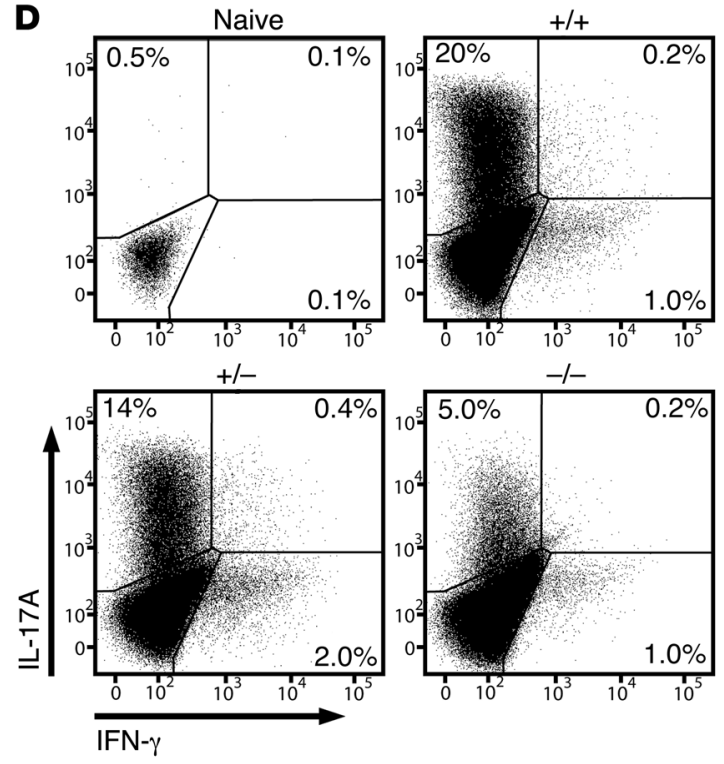

E

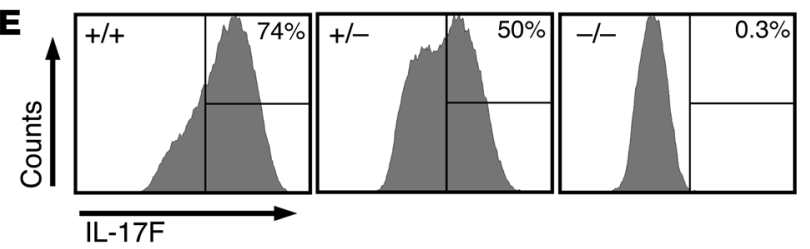

$\mathbf{F}$
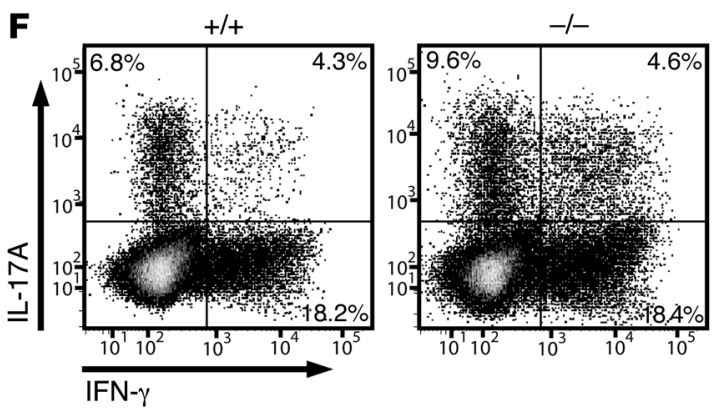

IL-6-driven Th cells were encephalitogenic, regardless of their secretion of IL-17A (30). Also, the strains of mice used appear to determine the therapeutic potential of anti-IL-17A therapy. While IL-17 blockade has hardly any effect in C57BL/6 mice (23), SJL/J mice show more pronounced disease alleviation after anti-IL-17A

\section{Figure 5}

Loss of IL-17F does not impact on T cell priming and does not lead to compensatory upregulation of IL-17A. (A-C) Mice were immunized with $\mathrm{MOG}_{35-55} / \mathrm{CFA}$ and lymphocytes were isolated from $\mathrm{LNs}$ prior to disease onset at 7 days after immunization. Cells were rechallenged with $50 \mu \mathrm{g} / \mathrm{ml}$ of $\mathrm{MOG}_{35-55}$, and IL-17A and IL-2 were measured by ELISA (A) and ELISPOT (B). (C) Proliferation of effector Th cells upon stimulation with $\mathrm{MOG}_{35-55}$ peptide or concanavalin $\mathrm{A}$ (ConA) was measured by thymidine incorporation. (A-C) A representative of 3 independent experiments is shown. Error bars indicate SEM of measured replicates. (D) Splenocytes from naive mice were polarized toward the Th17 effector type in vitro, and IL-17A, IL-17F, and IFN- $\gamma$ were measured by intracellular cytokine staining. Dot plots are gated on Th cells $\left(\mathrm{CD} 4^{+}\right)$, and histograms are gated on Th17 cells (CD4+, IL-17A $\left.{ }^{+}\right) .(E)$ Within the IL-17A-expressing Th17 compartment, IL-17F production is shown for each genotype. (F) CNS-infiltrating lymphocytes were isolated on day 24 , after immunization from severely sick mice, restimulated with PMA/ionomycin and Brefeldin A for 5 hours, and analyzed for their IL-17A and IFN- $\gamma$ expression by flow cytometry. (D-F) Percentages of gated cells are shown.

treatment (9). Nevertheless, neutralization of IL-17A has not been demonstrated to completely prevent EAE development. Kroenke et al. recently reported that both Th1 and Th17 cells have encephalitogenic potential (31), while O'Connor and colleagues demonstrated that Th1 cells are required to initiate CNS inflammation and Th17 cells invade the CNS only later during disease (32).

By now, a whole family of new cytokines has been grouped around IL-17A, all sharing a distinct structural feature, a 4-cysteine knot (33). The closest associate to IL-17A is $\operatorname{IL}-17 \mathrm{~F}(13,14)$. Both cytokines are functionally related to neutrophil recruitment and expansion, angiogenesis, tissue remodeling, and the induction of proinflammatory factors, like IL-1 $\beta$, TNF- $\alpha$, chemokines, and defensins $(11,34,35)$. We found that the loss of IL-17A coincided with consistently elevated levels of IL-17F expression, making IL-17F an ideal candidate to compensate for the loss of IL-17A in gene-targeted mice. We generated IL-17F-deficient mice and discovered that they are also fully susceptible to EAE and have no appreciable defect in generating an inflammatory response. One could argue conversely, that in Il17f/- mice, IL-17A could compensate and that the actual function of Il17a and Il17f can only be appreciated in mice lacking both genes. Unfortunately, the close proximity of the loci of these 2 cytokines makes it virtually impossible to obtain double-deficient mice by mere interbreeding (approximately 44-kb distance between the 2 genes). To prevent any potential compensatory effect of IL-17A in $I l 17 f^{-/}$mice, we treated mice with antagonistic anti-IL-17A mAb as used previously by Langrish et al. (9). The fact that Il17f $f^{-/}$mice treated with anti-IL-17A mAbs developed only a slightly ameliorated disease course, with a similar incidence and severity as untreated mice or WT mice, supports the notion that neither IL-17A nor IL-17F, either individually or in combination, are essential for the development of autoimmune CNS inflammation. In addition, the fact that mice in which transgenic overexpression of IL-17A is directed toward $\mathrm{T}$ cells also displayed an unaltered EAE phenotype further eliminates IL-17 as a key player in CNS autoimmunity. Integrating all current data generated by deletion of IL-17A as well as IL-17F and IL-22 (36) or induced overexpression of IL-17A by critical evaluation of its statistical versus biological relevance, we must conclude that, unlike IL-23, neither of these Th17 cytokines are key players in EAE. It is likely that the tissue distribution of 


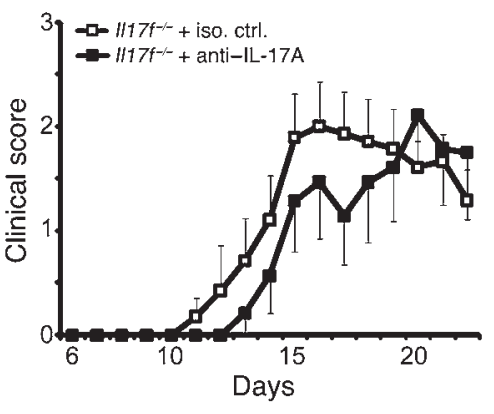

Figure 6

Loss of IL-17F and inhibition of IL-17A does not significantly impact on the development of EAE. EAE was induced in IL-17F-deficient mice by immunization with $\mathrm{MOG}_{35-55} / \mathrm{CFA}$. To antagonize IL-17A function, 200 $\mu \mathrm{g}$ of neutralizing anti-IL-17A mAb or the respective isotype control mAb was injected i.p. every fourth day, starting on day 4 after immunization. The graph shows the development of EAE according to clinical scores in 1 out of 2 independent experiments. Error bars represent mean \pm SEM. Iso. ctrl., isotype control.

IL-17RA and IL-17RC, the receptor complex for IL-17A and IL-17F $(17,18)$, determines the pathogenic capacity of Th17 cells. The CNS expresses low levels of IL-17R in comparison to the skin or lung $(37,38)$, thus explaining the severe impact of total IL-17A overexpression on the skin but not the CNS. Translating these findings into the clinic for therapeutic targeting of this pathway in MS is even more complicated considering the data obtained in a recent clinical trial in MS patients (39). MS patients were treated with $\mathrm{mAb}$ specific to IL-12/IL-23p40, which surprisingly had no beneficial effect, indicating that in MS patients, these Th1- and Th17-inducing cytokines may not play an essential role in disease progression. On the other hand, targeting of the IL-23 pathway has a profound efficacious impact on psoriasis, again supporting the notion that the tissue distribution of cytokine receptors such as IL-17R determines the impact of Th17 cells on inflammation.

The mere presence of a proinflammatory molecule at the inflammatory site is often interpreted to convey a vital function ultimately to be translated into an attractive drug target. However, while we can clearly confirm that IL-17A and IL-17F have all the features of such a factor, in vivo targeting shows that this correlation is not in fact causative. While IL-17 family member molecules currently serve their purpose as markers for pathogenic self-reactive cells, we conclude that other, thus far, unidentified factors or mechanisms employed by Th17 cells must convey their pathogenic capacity. Alternatively, it is also possible that Th17 or Th1 cells harbor a small population of IL-23-driven T cells, which possess all the pathogenic potential, and that IL-17 gained its encephalitogenic role based upon the concept of "guilt by association".

\section{Methods}

Mice. Female C57BL/6 mice were purchased from Harlan Laboratories. $I l 17 a^{-/-}$mice were generously provided by Yoichiro Iwakura (University of Tokyo, Tokyo, Japan). Il17f $f^{-/}\left(129 \times\right.$ C57BL/6) and IL-17A $\mathrm{A}^{\text {ind/+ }}$ mice were generated as described in Supplemental Figure 1G. Animal experiments were approved by the Swiss Veterinary Office (Zurich, Switzerland) and the Central Animal Facility Institution of the University of Mainz.

Induction of EAE. For EAE induction, mice were immunized subcutaneously, with $200 \mu \mathrm{g}$ of $\mathrm{MOG}_{35-55}$-peptide emulsified in complete Freund's adjuvant supplemented with $2 \mathrm{mg} / \mathrm{ml}$ of Mycobacterium tuberculosis, into the lateral abdomen. On days 0 and 2 after immunization, $200 \mu \mathrm{g}$ of pertussis toxin and PBS was administered intraperitoneally. Clinical disease was scored daily as follows: 0 , no clinical disease; 1 , limp tail; 2 , impaired righting reflex; 3, hind limb paralysis; 4, moribund; 5, dead (4).

In vitro assays. Lymphocytes were cultured in RPMI1640 containing 10\% FCS (both from Invitrogen). Restimulation of primed lymphocytes from $\mathrm{LN}$, spleen, or cerebellum was measured at 7 days after immunization (preEAE) by $\mathrm{MOG}_{35-55} / \mathrm{CFA}$ (4) and challenged with $50 \mu \mathrm{g} / \mathrm{ml} \mathrm{MOG}_{35-55}$ or $5 \mu \mathrm{g} / \mathrm{ml}$ concanavalin A. Proliferation was assessed by thymidine incorporation (40) and cytokine release by either FlowCytomix Th1/2 Multiplex (Bender MedSystems), ELISA (BD Biosciences - Pharmingen), or ELISPOT (2) as described. Activating anti-CD3 and anti-CD28 antibodies were used at concentrations of $1 \mu \mathrm{g} / \mathrm{ml}$ and $6 \mathrm{ng} / \mathrm{ml}$, respectively. Fluorocytometric analysis of surface marker expression was performed as described (41). Intracellular cytokine staining was performed with the Cytofix/Cytoperm Plus Kit (BD Bioscience), according to manufacturers directions. The following antibodies were used: anti-IL-17A (TC11-18H10; BioLegend), anti-IFN- $\gamma$ (XMG1.2; BD Biosciences - Pharmingen), and anti-IL-17F (R\&D Systems). The IL-17F antibody was labeled using the Alexa Fluor 488 Monoclonal Antibody Labeling Kit (Invitrogen).

T cell polarization. For the in vitro generation of Th17 cells, splenocytes were harvested 7 days after immunization and were restimulated with $20 \mu \mathrm{g} / \mathrm{ml} \mathrm{MOG}_{35-55}$ (BioExpress), $5 \mathrm{ng} / \mathrm{ml}$ TGF- $\beta$, $20 \mathrm{ng} / \mathrm{ml} \mathrm{IL-6} \mathrm{(both}$ from PeproTech EC), $10 \mathrm{ng} / \mathrm{ml}$ IL-23 (R\&D Systems), $5 \mu \mathrm{g} / \mathrm{ml}$ anti-IFN- $\gamma$ (R4-6A2; Bioexpress), and $5 \mathrm{ng} / \mathrm{ml} \mathrm{IL-2} \mathrm{(eBioscience).} \mathrm{Anti-IFN-} \gamma$ was added daily, and IL-23 was added on days 0 and 2. Cells were analyzed on day 6 after culture.

Real-time RT-PCR. RNA was extracted and cDNA prepared as described. The primers used for IL-17F were as follows: forward, CTGTTGATGTTGGGACTTGCC, and reverse, TCACAGTGTTATCCTCCAGG. $\beta$-actin and IL-17A primers were described elsewhere (2).

Histology and immunobistochemical staining. Spinal columns were fixed in 4\% paraformaldehyde in PBS, paraffin embedded, cut, and stained with $\mathrm{H} \& \mathrm{E}$, according to standard protocols. Immunohistochemical stainings on serial sections using antibodies to CNPase (1:500; Chemicon) and glial fibrillary acidic protein (1:4,000; DAKO) were carried out on an automated BenchMark Staining apparatus (Ventana Medical Systems), following the manufacturer's guidelines.

Extraction of mononucleated cells from inflamed CNS tissue and subsequent cytofluorometric analysis was performed as described previously (41).

Statistics. Clinical development was evaluated using a 2-tailed Student's $t$ test as well as analysis of covariance analysis, using the R project for statistical computing. $P$ values of less than 0.05 were considered significant.

\section{Acknowledgments}

The work was supported by grants from Swiss National Science Foundation (to B. Becher), US National MS Society (to B. Becher), Swiss MS Society (to B. Becher), National Center of Competence in Research (NCCR-Neuro) (to B. Becher), and an unrestricted grant from Merck Serono International (to B. Becher); FP6 Marie Curie Research Training Network (IMDEMI) (MRTN-CT-2004-005632, to A. Waisman), Deutsche Forschungsgemeinschaft (SFB490 and $\mathrm{SFB} / \mathrm{TR} 52$, to A. Waisman), and funds from Boehringer Ingelheim Stiftung (to A. Waisman); and US NIH (NINDS R01 NS046006; to F.L. Heppner). We thank Y. Iwakura for providing us with his IL-17A-deficient mouse strain. We also thank V. Wörtmann, M. Perkovic, M. Snetkova, and E. Wiese for excellent technical assistance and C. Jakobsen, P. von Landenberg, T. Buch, J. vom Berg, T. Wunderlich, J. Petermann, F. Frommer, F. Kurschus, N. Yogev, S. Wörtge, and $\mathrm{K}$. Reifenberg for advice, critical comments, and discussions. 
Received for publication April 22, 2008, and accepted in revised form November 5, 2008.

Address correspondence to: Burkhard Becher, University of Zurich, University Hospital, Division of Neuroimmunology, Institute of Experimental Immunology, Department of Pathology,
Winterthurer Strasse 190, CH-8057 Zürich, Switzerland. Phone: 41-44-635-3703; Fax: 41-44-635-6883; E-mail: burkhard.becher@ neuroimm.uzh.ch. Or to: Ari Waisman, First Medical Department, University of Mainz, Obere-Zahlbacherstr. 63, D-55131 Mainz, Germany. Phone: 49-6131-3933357; Fax: 49-6131-3932104; E-mail: waisman@uni-mainz.de.
1. McFarland, H.F., and Martin, R. 2007. Multiple sclerosis: a complicated picture of autoimmunity. Nat. Immunol. 8:913-919.

2. Gutcher, I., and Becher, B. 2007. APC-derived cytokines and $\mathrm{T}$ cell polarization in autoimmune inflammation. J. Clin. Invest. 117:1119-1127.

3. Becher, B., Durell, B.G., and Noelle, R.J. 2002. Experimental autoimmune encephalitis and inflammation in the absence of interleukin-12. J. Clin. Invest. 110:493-497.

4. Gutcher, I., Urich, E., Wolter, K., Prinz, M., and Becher, B. 2006. Interleukin 18-independent engagement of interleukin 18 receptor-alpha is required for autoimmune inflammation. Nat. Immunol. 7:946-953.

5. Willenborg, D.O., Fordham, S., Bernard, C.C., Cowden, W.B., and Ramshaw, I.A. 1996. IFNgamma plays a critical down-regulatory role in the induction and effector phase of myelin oligodendrocyte glycoprotein-induced autoimmune encephalomyelitis. J. Immunol. 157:3223-3227.

6. Ferber, I.A., et al. 1996. Mice with a disrupted IFN-gamma gene are susceptible to the induction of experimental autoimmune encephalomyelitis (EAE). J. Immunol. 156:5-7.

7. Cua, D.J., et al. 2003. Interleukin-23 rather than interleukin-12 is the critical cytokine for autoimmune inflammation of the brain. Nature. 421:744-748.

8. Bettelli, E., Oukka, M., and Kuchroo, V.K. 2007. $\mathrm{T}-\mathrm{H}-17$ cells in the circle of immunity and autoimmunity. Nat. Immunol. 8:345-350.

9. Langrish, C.L., et al. 2005. IL-23 drives a pathogenic $T$ cell population that induces autoimmune inflammation. J. Exp. Med. 201:233-240.

10. Veldhoen, M., Hocking, R.J., Atkins, C.J., Locksley, R.M., and Stockinger, B. 2006. TGFbeta in the context of an inflammatory cytokine milieu supports de novo differentiation of IL-17-producing T cells. Immunity. 24:179-189.

11. Weaver, C.T., Hatton, R.D., Mangan, P.R., and Harrington, L.E. 2007. IL-17 family cytokines and the expanding diversity of effector $\mathrm{T}$ cell lineages. Annu. Rev. Immunol. 25:821-852.

12. Steinman, L. 2008. A rush to judgment on Th17. J. Exp. Med. 205:1517-1522.

13. Starnes, T., et al. 2001. Cutting edge: IL-17F, a novel cytokine selectively expressed in activated $\mathrm{T}$ cells and monocytes, regulates angiogenesis and endothelial cell cytokine production. J. Immunol. 167:4137-4140.

14. Kawaguchi, M., et al. 2001. Identification of a novel cytokine, ML-1, and its expression in subjects with asthma. J. Immunol. 167:4430-4435.

15. Moseley, T.A., Haudenschild, D.R., Rose, L., and Reddi, A.H. 2003. Interleukin-17 family and IL-17 receptors. Cytokine Growth Factor Rev. 14:155-174.

16. Liu, S.J., et al. 2007. Induction of a distinct CD8 Tnc17 subset by transforming growth factor-beta and interleukin-6. J. Leukoc. Biol. 82:354-360.

17. Toy, D., et al. 2006. Cutting edge: interleukin 17 signals through a heteromeric receptor complex. J. Immunol. 177:36-39.

18. Wright, J.F., et al. 2008. The human IL-17F/ IL-17A heterodimeric cytokine signals through the IL-17RA/IL-17RC receptor complex. J. Immunol. 181:2799-2805.

19. Kolls, J.K., and Linden, A. 2004. Interleukin-17 family members and inflammation. Immunity. 21:467-476.

20. Schwenk, F., Baron, U., and Rajewsky, K. 1995. A cre-transgenic mouse strain for the ubiquitous deletion of loxP-flanked gene segments including deletion in germ cells. Nucleic Acids Res. 23:5080-5081.

21. Komiyama, Y., et al. 2006. IL-17 plays an important role in the development of experimental autoimmune encephalomyelitis. J. Immunol. 177:566-573.

22. Yang, X.O., et al. 2008. Regulation of inflammatory responses by IL-17F. J. Exp. Med. 205:1063-1075.

23. Hofstetter, H.H., et al. 2005. Therapeutic efficacy of IL-17 neutralization in murine experimental autoimmune encephalomyelitis. Cell Immunol. 237:123-130.

24. Mosmann, T.R., and Coffman, R.L. 1989. TH1 and TH2 cells: different patterns of lymphokine secretion lead to different functional properties. Annu. Rev. Immunol. 7:145-173.

25. Frei, K., et al. 1997. Tumor necrosis factor alpha and lymphotoxin alpha are not required for induction of acute experimental autoimmune encephalomyelitis. J. Exp. Med. 185:2177-2182.

26. Kebir, H., et al. 2007. Human TH17 lymphocytes promote blood-brain barrier disruption and central nervous system inflammation. Nat. Med. 13:1173-1175.

27. Rohn, T.A., et al. 2006. Vaccination against IL-17 suppresses autoimmune arthritis and encephalomyelitis. Eur. J. Immunol. 36:2857-2867.

28. Yoshimura, T., et al. 2008. Differential roles for IFN-gamma and IL-17 in experimental autoimmune uveoretinitis. Int. Immunol. 20:209-214.

29. Luger, D., et al. 2008. Either a Th17 or a Th1 effector response can drive autoimmunity: conditions of disease induction affect dominant effector cat- egory. J. Exp. Med. 205:799-810.

30. McGeachy, M.J., et al. 2007. TGF-beta and IL-6 drive the production of IL-17 and IL-10 by T cells and restrain $\mathrm{T}(\mathrm{H})-17$ cell-mediated pathology. Nat. Immunol. 8:1390-1397.

31. Kroenke, M.A., Carlson, T.J., Andjelkovic, A.V., and Segal, B.M. 2008. IL-12- and IL-23-modulated $\mathrm{T}$ cells induce distinct types of EAE based on histology, CNS chemokine profile, and response to cytokine inhibition. J. Exp. Med. 205:1535-1541.

32. O'Connor, R.A., et al. 2008. Cutting edge: Th1 cells facilitate the entry of Th17 cells to the central nervous system during experimental autoimmune encephalomyelitis. J. Immunol. 181:3750-3754.

33. Hymowitz, S.G., et al. 2001. IL-17s adopt a cystine knot fold: structure and activity of a novel cytokine, IL-17F, and implications for receptor binding. ЕМВО J. 20:5332-5341.

34. Liang, S.C., et al. 2006. Interleukin (IL)-22 and IL-17 are coexpressed by Th17 cells and cooperatively enhance expression of antimicrobial peptides. J. Exp. Med. 203:2271-2279.

35. Ye, P., et al. 2001. Requirement of interleukin 17 receptor signaling for lung $\mathrm{CXC}$ chemokine and granulocyte colony-stimulating factor expression, neutrophil recruitment, and host defense. J. Exp. Med. 194:519-527.

36. Kreymborg, K., et al. 2007. IL-22 is expressed by TH17 cells in an IL-23-dependent fashion, but not required for the development of autoimmune encephalomyelitis. J. Immunol. 179:8098-8104.

37. Haudenschild, D., Moseley, T., Rose, L., and Reddi, A.H. 2002. Soluble and transmembrane Isoforms of novel interleukin-17 receptor-like protein by RNA splicing and expression in prostate cancer. J. Biol. Chem. 277:4309-4316.

38. Yao, Z.B., et al. 1995. Herpesvirus Saimiri encodes a new cytokine, IL-17, which binds to a novel cytokine receptor. Immunity. 3:811-821.

39. Segal, B.M., et al. 2008. Repeated subcutaneous injections of IL12/23 p 40 neutralising antibody, ustekinumab, in patients with relapsing-remitting multiple sclerosis: a phase II, double-blind, placebo-controlled, randomised, dose-ranging study. Lancet Neurol. 7:796-804.

40. Heppner, F.L., et al. 2005. Experimental autoimmune encephalomyelitis repressed by microglial paralysis. Nat. Med. 11:146-152.

41. Valenzuela, D.M., et al. 2003. High-throughput engineering of the mouse genome coupled with high-resolution expression analysis. Nat. Biotechnol. 21:652-659. 